\title{
Assembly of a novel biosynthetic pathway for gentamicin B production in Micromonospora echinospora
}

Xianpu Ni, Zhenpeng Sun, Yawen Gu, Hao Cui and Huanzhang Xia*

\begin{abstract}
Background: Isepamicin is a weakly toxic but highly active aminoglycoside antibiotic derivative of gentamicin $\mathrm{B}$. Gentamicin B is a naturally occurring minor component isolated from Micromonospora echinospora. 2'- $\mathrm{NH}_{2}$-containing gentamicin C complex is a dominant compound produced by wild-type M. echinospora; by contrast, 2'-OHcontaining gentamicin $B$ is produced as a minor component. However, the biosynthetic pathway of gentamicin $B$ remains unclear. Considering that gentamicin $B$ shares a unique $C_{2}$, hydroxyl group with kanamycin $A$, we aimed to design a new biosynthetic pathway of gentamicin $B$ by combining twelve steps of gentamicin biosynthesis and two steps of kanamycin biosynthesis.
\end{abstract}

Results: We blocked the biosynthetic pathway of byproducts and generated a strain overproducing Jl-20A, which was used as a precursor of gentamicin B biosynthesis, by disrupting genK and genP. The amount of JI-20A produced in M. echinospora $\triangle K \Delta \mathrm{P}$ reached $911 \mathrm{\mu g} / \mathrm{ml}$, which was 14-fold higher than that of M. echinospora $\Delta \mathrm{P}$. The enzymes KanJ and KanK necessary to convert 2'- $\mathrm{NH}_{2}$ into 2'-OH from the kanamycin biosynthetic pathway were heterologously expressed in M. echinospora $\triangle K \triangle P$ to transform JI-20A into gentamicin B. The strain with kanJK under PermE* could produce $80 \mu \mathrm{g} / \mathrm{ml}$ of gentamicin B, which was tenfold higher than that of the wild-type strain. To enhance gentamicin B production, we employed different promoters and gene integration combinations. When a PhrdB promoter was used and kanJ and kanK were integrated in the genome through gene replacement, gentamicin B was generated as the major product with a maximum yield of $880 \mu \mathrm{g} / \mathrm{ml}$.

Conclusion: We constructed a new biosynthetic pathway of high-level gentamicin B production; in this pathway, most byproducts were removed. This method also provided novel insights into the biosynthesis of secondary metabolites via the combinatorial biosynthesis.

Keywords: Micromonospora echinospora, Gentamicin B, Metabolic engineering, Artificial biosynthetic pathway

\section{Background}

Aminoglycoside antibiotics have been widely used to treat severe bacterial infections; for instance, streptomycin was administered as the first effective anti-tuberculosis agent. Other aminoglycoside antibiotics include 2-deoxystreptamine (2-DOS) containing gentamicin, kanamycin, neomycin, and butirosin. However, the critical resistance mechanism of aminoglycoside antibiotics in pathogens is enzymatic inactivation. As such,

*Correspondence: xiahz612@sina.com

School of Life Science and Biopharmaceutics, Shenyang Pharmaceutical University, No.103 Wenhua Road, Shenyang, Liaoning, China semi-synthetic aminoglycosides have been created to overcome pathogen enzymatic inactivation. For example, isepamicin is developed by introducing (S)-3-amino2-hydroxypropionyl side chains to the 1-amino group of gentamicin B. This side chain can block the modification by various aminoglycoside-modifying enzymes. Thus, isepamicin is highly stable against aminoglycoside-inactivating enzymes [1].

Isepamicin is manufactured from gentamicin $\mathrm{B}$, which is co-produced in Micromonospora echinospora. Gentamicin $\mathrm{C}$ complex is produced by $M$. echinospora as its major product. By contrast, gentamicin $\mathrm{A}, \mathrm{B}$, and $\mathrm{X}$ are 
yielded by $M$. echinospora as minor components [2]. Gentamicin A and X are intermediates of gentamicin C biosynthesis. However, gentamicin $\mathrm{B}$ is not an intermediate of the gentamicin $C$ biosynthetic pathway because gentamicin B cannot be biotransformed by M. echinospora into any gentamicin $\mathrm{C}$ component [2]. Thus far, the biosynthetic pathway of gentamicin $\mathrm{B}$ remains unclear.

The biosynthetic pathway of gentamicin has been elucidated (Fig. 1). The gene cluster of gentamicin has also been cloned [3, 4]. The genes involved in the biosynthesis of pseudodisaccharide paromamine and pseudotrisaccharide gentamicin A2 have been identified [5]. The production of gentamicin X2 from A2 involves four enzymes: oxidoreductase, transaminase, and two methyltransferases [6]. X2 can also be transformed into JI-20A by C-6 dehydrogenase and transaminase [7-9]. Moreover, GenK is the methyltransferase of $\mathrm{C}^{\prime} 6^{\prime}$ in gentamicin, and gen $\mathrm{K}$ disruption generates a gentamicin C1a-overproducing strain [10-12]. GenP is a phosphotransferase participating in $3^{\prime}, 4^{\prime}$-deoxygenation in the gentamicin biosynthetic pathway [13]. If genP is disrupted, JI-20A,JI-20B, and JI-20Ba accumulate in mutant strains [9]. In this study, gen $\mathrm{K}$ and gen $\mathrm{P}$ were disrupted simultaneously to generate strains producing JI-20A, which was designed as a precursor of gentamicin B biosynthesis (Fig. 1).

The structure of gentamicin $B$ resembles that of both gentamicin $\mathrm{C}$ and kanamycin A. Unlike most 2-DOScontaining aminoglycosides, kanamycin A contains a hydroxy group at $C_{2}$; gentamicin $B$ shares this unique structure with kanamycin $\mathrm{A}$. The methylated pentose ring of gentamicin $\mathrm{B}$ is also similar to that of gentamicin $\mathrm{C}$ and is designated as garaosamine. Gentamicin B can be synthesized via a biosynthetic pathway similar to that of kanamycin A on the basis of molecular structure. In the kanamycin A biosynthetic pathway in Streptomyces kanamyceticus, 2'- $\mathrm{NH}_{2}$ is converted into 2'-OH by using $\mathrm{KanJ}$ and KanK; in the process, 2-oxoglutarate, $\mathrm{Fe}^{2+}, \mathrm{O}_{2}$, and NADH are utilized [14]. We hypothesized that gentamicin B could be biosynthesized from JI-20A, a compound structurally similar to gentamicin $\mathrm{B}$, except $\mathrm{NH}_{2}$ group at $\mathrm{C}_{2^{\prime}}$ (Fig. 1). Thus, JI-20A could be converted into gentamicin B in JI-20A-overproducing strain via two steps catalyzed by KanJ and KanK. Using the assembly and metabolically engineered biosynthesis pathway of gentamicin B, we can eliminate byproducts and improve gentamicin B production.

\section{Results and discussion}

\section{Construction of $\mathrm{Jl}-20 \mathrm{~A}$-overproducing strain by disrupting} gen $\mathrm{P}$ and gen K genes

The original strain can potentially generate gentamicin $B$; as such, we determined whether gentamicin B is synthesized from the 2'-amino-containing precursor by the KanJ and KanK homologs. However, the genes homologous to kanJ and kanK have yet to be detected in M. echinospora. Considering that the biosynthetic pathway of gentamicin B remains unclear, we aimed to construct an artificial pathway of gentamicin B biosynthesis. JI-20A and gentamicin B are similar in terms of chemical structure except at C2'. JI-20A contains an amino group at $\mathrm{C}^{\prime}$, whereas gentamicin B comprises a hydroxyl group at the same position. The structural difference between JI-20A and gentamicin B is similar to the difference between kanamycin A and B. Considering that kanamycin B is converted into kanamycin A by KanJ and KanK, we determined whether KanJ and KanK can also transform JI-20A into gentamicin B.

To generate a JI-20A-producing strain, we simultaneously disrupted the $6^{\prime}$-methyltransferase gene genK and the 3'-phosphotransferase gene gen $\mathrm{P}$ in this study (Fig. 2). We separately generated gen $\mathrm{P}$ - and gen $\mathrm{K}$-disrupting strains in our previous work [6, 7]. The genP-disrupting strain does not produce gentamicin $C$ complex, which are the main products of the wild-type strain but are the main byproducts in gentamicin B production. In this study, the genK-disrupting plasmid was introduced to M. echinospora $\Delta \mathrm{P}$ to obtain the double-gene-disrupting strain $M$. echinospora $\Delta \mathrm{K} \Delta \mathrm{P}$ (Additional file 1: Figure $\mathrm{S} 1 \mathrm{~A})$. The disrupting strain was fermented and its products were analyzed through high-performance liquid chromatography with evaporative light scattering detector (HPLC-ELSD). JI-20B and JI-20Ba were the main products of $M$. echinospora $\Delta \mathrm{P}$ but were undetectable in M. echinospora $\Delta \mathrm{K} \Delta \mathrm{P}$ (Fig. 3). JI-20A was produced up to $911 \mu \mathrm{g} / \mathrm{ml}$, which was 14 -fold higher than that produced by $M$. echinospora $\Delta \mathrm{P}$. We blocked the biosynthesis of gentamicin C1, C1a, C2, C2a, JI-20B, and JI-20Ba and generated a JI-20A-overproducing strain by disrupting gen $\mathrm{P}$ and $g e n \mathrm{~K}$.

\section{Construction of a new gentamicin B biosynthetic pathway through the heterologous expression of kanJ and kanK}

The kan $\mathrm{J}$ and $k a n \mathrm{~K}$ genes under the control of the strong promoter PermE* were cloned into the site-specific integration plasmid pEAP1 to construct pSPUJK1. pSPUJK1 was introduced to $M$. echinospora $\triangle \mathrm{K} \Delta \mathrm{P}$ through conjugation and exconjugants were selected through erythromycin resistance; as a result, $M$. echinospora JK1 was generated (Additional file 1: Figure S1B). M. echinospora JK1 was fermented under the same condition used to ferment the wild-type strain. The fermentation products of M. echinospora JK1 were also analyzed through HPLCELSD. Compared with the wild-type strain, $M$. echinospora JK1 produced a new product exhibiting a retention time similar to that of gentamicin B (Fig. 3). 


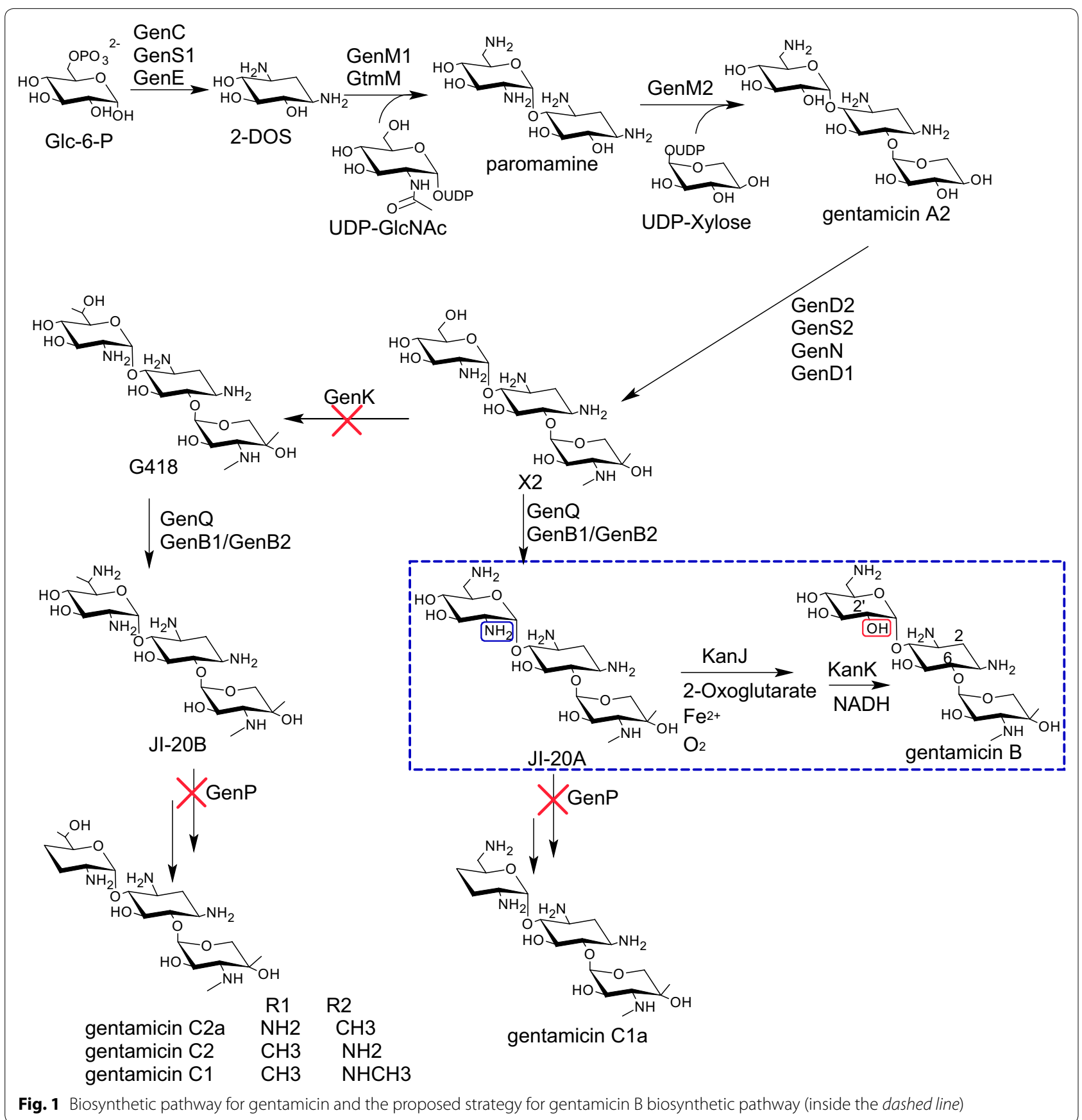

To determine the structure of the new product of $M$. echinospora $\mathrm{JK} 1$, we analyzed the purified product through mass spectrometry. The exact mass of the product was $482.26(\mathrm{~m} / \mathrm{z} 483.26)$, which corresponds to gentamicin B (Fig. 4). Furthermore, the protonated fragments were the same as those of the reported mass spectra of gentamicin B [15], that is, the glycoside bond of gentamicin B cleavage formed the fragment $\mathrm{b}+\mathrm{c}$ $(m / z 324.17)$. The glycoside found at the $\mathrm{C}_{6}-\mathrm{O}$ of 2-DOS decomposed; thus, the fragments $\mathrm{b}+\mathrm{c}+\mathrm{x}(\mathrm{m} / z$ 366.18) were produced. Nevertheless, gentamicin $B$ yields the same molecular weight as that of gentamicin X2, which is another minor component of gentamicin. To verify the structure of the new product, we recorded the corresponding ${ }^{1} \mathrm{H}$ and ${ }^{13} \mathrm{C}$ NMR data. The ${ }^{1} \mathrm{H}$ spectrum of the new product was similar to that of gentamicin $\mathrm{B}$ [16] (Additional file 2: Figure S2). Moreover, the ${ }^{13} \mathrm{C}$ NMR data of the new product (Table 1) were identical with the 

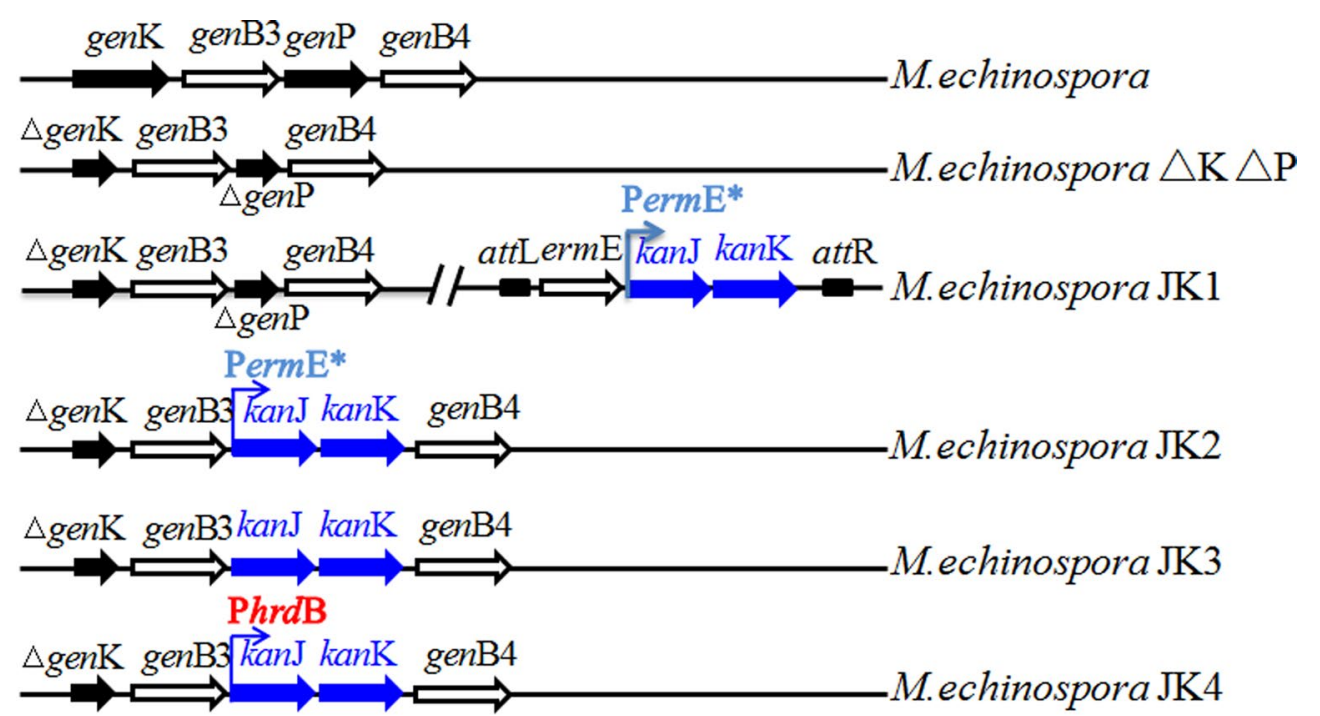

Fig. 2 Genotypes of M. echinospora and its recombinant strains

reported NMR data of gentamicin B [17]. MS and NMR data demonstrated that the product from M. echinospora JK1 is gentamicin B.

Although gentamicin B production is greatly improved in $M$. echinospora JK1 compared with that of the wildtype strain, the yield is only $85 \mu \mathrm{g} / \mathrm{ml}$ in $M$. echinospora JK1. A large quantity of JI-20A in M. echinospora JK1 was also found in the fermentation broth. Therefore, the expression levels of $k a n \mathrm{~J}$ and $k a n \mathrm{~K}$ were genetically manipulated to improve gentamicin B production.

\section{Enhancing gentamicin B production through the genetic manipulation of kanJK}

The genetic stability of the strains applied in industrial fermentation is very important. However, gentamicin B production in $M$. echinospora JK1 decreased from $162 \mu \mathrm{g} / \mathrm{ml}$ to $80 \mu \mathrm{g} / \mathrm{ml}$ in five generations of unselected passages (Fig. 5a). We proposed that the instability of $M$. echinospora $\mathrm{JK} 1$ is caused by the homologous recombination between the PermE* upstream of kanJK and the native promoter of ermE, which was used as a selection marker gene in pSPUJK1. In addition, the instability may be caused by chromosomal rearrangements or plasmid elimination from the chromosome, which can occur when a $\phi C 31$-derived plasmid is used in strains containing multiple pseudo attB sites [18].

To avoid genetic instability, we integrated kanJ and kanK into the chromosome through homologous recombination instead of site-specific insertion. Considering that gen $\mathrm{P}$ has been disrupted in $M$. echinospora $\Delta \mathrm{K} \Delta \mathrm{P}$, we designed $k a n \mathrm{~J}$ and $k a n \mathrm{~K}$ to replace gen P (Additional file 1: Figure S1C). To reduce JI-20A production and improve gentamicin B production, we placed kanJ and $k a n \mathrm{~K}$ under the control of the strong promoter PermE* (Fig. 2). Moreover, the fermentation broth of the gene replacement strain $M$. echinospora JK2 was analyzed through HPLC-ELSD. Figure 5a shows that the gentamicin B production by $M$. echinospora JK2 was more stable than that by $M$. echinospora JK1. Gentamicin B production increased to $342 \mu \mathrm{g} / \mathrm{ml}$. However, M. echinospora JK2 produced a considerable quantity of JI-20A.

The native promoter of gen $\mathrm{P}$ was used to improve gentamicin $\mathrm{B}$ production. To eliminate any possibility of polar effects on other genes, we designed kanJK as a replacement of the open reading frame of genP, and no other elements were introduced (Additional file 1: Figure S1D). As such, the native ribosome binding site (RBS) and promoter of gen $\mathrm{P}$ (or promoter of its operon) were employed by kanJK. Although the regulatory elements of genP have yet to be determined, we proposed that all regulatory elements of genP can be used. In the fermentation of $M$. echinospora JK3, kan JK was expressed as gentamicin B was produced in JK3. Gentamicin B production also reached $436 \mu \mathrm{g} / \mathrm{ml}$. PermE*, which exhibits a strong promoter activity, is widely used for the gene expression in Actinomyces. However, this work found that PermE* is weaker than the native promoter of genP.This phenomenon occurred possibly because PermE* originates from Saccharopolyspora erythraea; as such, PermE* cannot be as effective in M. echinospora as in S. erythraea. Another possible cause is the ineffectiveness of the RBS of the construction because the translation initiation of PermE*-kanJ-kanK is weak when analyzed with the RBS Calculator [19]. 


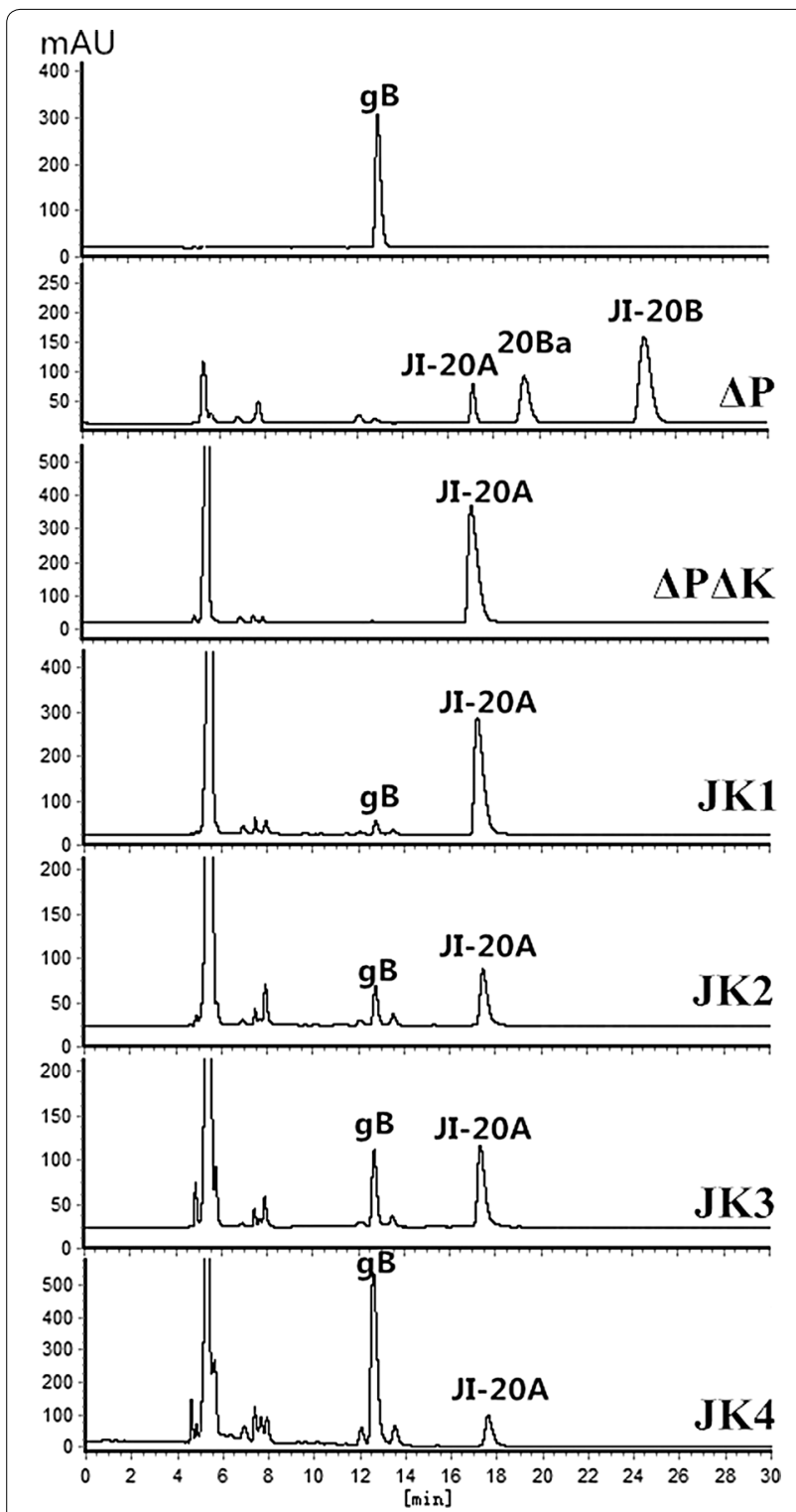

Fig. 3 HPLC-ELSD analysis of gentamicin production in fermentations. gB gentamicin B, 20Ba Jl-20Ba

JI-20A accumulation in the fermentation broths of $M$. echinospora JK2 and JK3 indicated the inefficiency of KanJ and KanK. Thus, an increase in KanJK expression may enhance their total activity and gentamicin B production. The PhrdB promoter of Streptomyces coelicoloris stronger than PermE* [20]. Therefore, a kanJK under the control of $\mathrm{PhrdB}$ was constructed and introduced to $M$. echinospora $\Delta \mathrm{K} \Delta \mathrm{P}$; as a result, a mutant strain $M$. echinospora JK4 was generated (Additional file 1: Figure S1E).

The HPLC analysis of the fermentation broth of $M$. echinospora JK4 revealed that gentamicin B production reached $880 \mu \mathrm{g} / \mathrm{ml}$; by contrast, JI-20A production decreased to $143 \mu \mathrm{g} / \mathrm{ml}$. Figure $5 \mathrm{~b}$ shows that gentamicin $\mathrm{B}$ production increased by 2.5 -fold when the $\mathrm{PhrdB}$ promoter was used compared with that when PermE* was used. This result confirmed that the expression level of KanJK was enhanced by replacing the promoter PermE* with PhrdB.

The genome analysis of Actinomyces revealed the presence of numerous gene clusters encoding secondary metabolites [21]. Actinomyces can be metabolically engineered to overproduce native metabolites or analogs. With the development of synthetic biological tools, improved bioinformatics tools of metabolic engineering, and enhanced sensitivity and sophistication of analytical methods, secondary metabolite production is feasible in various cellular factories [22].

\section{Conclusions}

We successfully established an artificial biosynthetic pathway to achieve a high-level production of gentamicin $\mathrm{B}$. The genes gen $\mathrm{K}$ and gen $\mathrm{P}$ were disrupted in $M$. echinospora to producethe JI-20A, which is the precursor of gentamicin B. JI-20Aproduction in the gene-disrupting strain $M$. echinospora $\Delta \mathrm{K} \Delta \mathrm{P}$ reached $911 \mu \mathrm{g} / \mathrm{ml}$, which was 14-fold higher than that of $M$. echinospora $\Delta \mathrm{P}$. We disrupted the biosynthesis of gentamicin C1, C1a, C2, $\mathrm{C} 2 \mathrm{a}, \mathrm{JI}-20 \mathrm{~B}$, and JI-20Ba by disrupting gen $\mathrm{P}$ and gen $\mathrm{K}$. The removal of byproducts in fermentation broth will be beneficial to purification because antibiotic purification is costly and time consuming.

An artificial pathway for the conversion of JI-20A to gentamicin B was constructed through the heterologous overexpression of kan J and kanK in M. echinospora $\Delta \mathrm{K} \Delta \mathrm{P}$. The kanJK-overexpressing strain under the control of PermE* can produce $80 \mu \mathrm{g} / \mathrm{ml}$ gentamicin B, which was tenfold higher than that of the wild-type strain. Different promoters and gene integration combinations were investigated to improve gentamicin B production. When the PhrdB promoter was used and kanJ and kanK were integrated in the genome through gene replacement, gentamicin B was produced as a major product with a maximum yield of $880 \mu \mathrm{g} / \mathrm{ml}$.These results confirmed that microbiological strains can be engineered through the metabolic engineering of an intrinsic biosynthetic pathway and the introduction of exogenous genes to produce high yields of target products.

\section{Methods}

Bacterial strains, plasmids, media, and culture conditions

The strains and plasmids used in this work are listed in Table 2. Escherichia coli Top10 was used as the cloning host grown on Luria-Bertani (LB) liquid or solid medium. Liquid ATCC172 was used for the vegetative growth of $M$. echinospora. The conjugal transfer was 


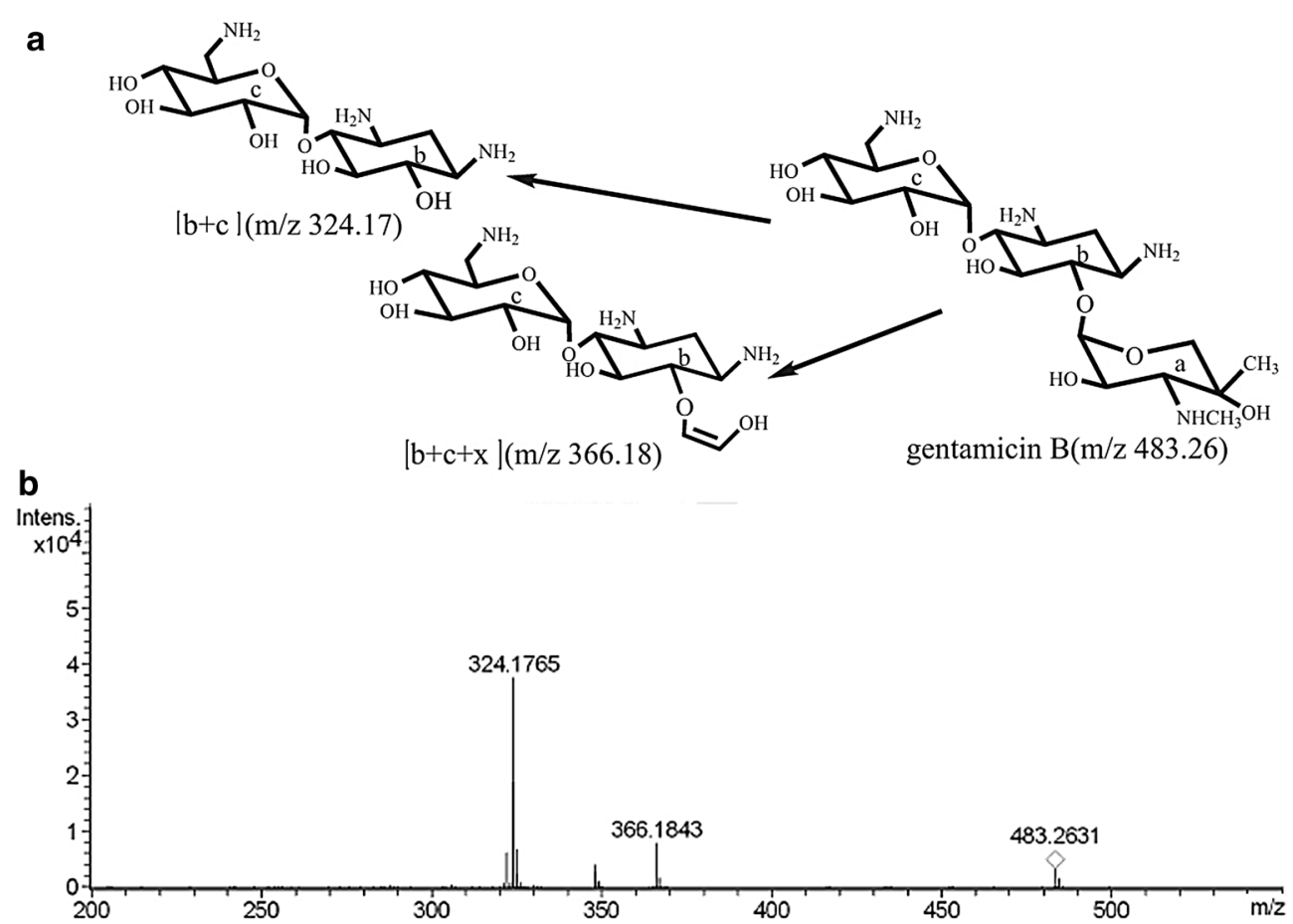

Fig. 4 Structural determination of gentamicin B by MS/MS. a Structure of gentamicin B. b Mass spectra of the new product from M. echinosporaJK1

Table $1{ }^{13} \mathrm{C}$ NMR spectral data for gentamicin B [16] and the new compound from M. echinospora JK1 (for atom numbering see Fig. 1)

\begin{tabular}{lcc}
\hline Atom & Gentamicin B ( $\mathbf{\delta}$ & New compound $(\boldsymbol{\delta})$ \\
\hline 1 & 50.6 & 49.7 \\
2 & 28.3 & 27.5 \\
3 & 48.4 & 47.5 \\
4 & 79.0 & 78.3 \\
5 & 73.1 & 72.3 \\
6 & 84.6 & 83.8 \\
$1^{\prime}$ & 96.6 & 95.9 \\
$2^{\prime}$ & 71.7 & 70.6 \\
$3^{\prime}$ & 73.0 & 72.0 \\
$4^{\prime}$ & 71.6 & 69.8 \\
$5^{\prime}$ & 69.5 & 68.6 \\
$6^{\prime}$ & 41.1 & 40.1 \\
$1^{\prime \prime}$ & 102.0 & 101.2 \\
$2^{\prime \prime}$ & 67.2 & 67.6 \\
$3^{\prime \prime}$ & 64.2 & 63.3 \\
$4^{\prime \prime}$ & 70.8 & 70.6 \\
$5^{\prime \prime}$ & 68.6 & 66.2 \\
$3^{\prime \prime}-\mathrm{N}-\mathrm{CH}_{3}$ & 35.3 & 34.3 \\
$4^{\prime \prime}-\mathrm{CH}_{3}$ & 21.7 & 20.8 \\
\hline
\end{tabular}

performed on MS agar. Solid slanting medium was used for $M$. echinospora sporulation. The previously described media and culture conditions were used for gentamicin production [8].

\section{Construction of kanJ and kanK expression plasmids}

DNA isolation and manipulation were performed as described by Sambrook [23]. Additional file 3: Table S1 lists the primers used in this work. The kanJ and kanK genes were amplified from the genomic DNA of S. kanamyceticus. The primers were designed using the biosynthetic gene sequence of kanamycin (GenBank accession number: AJ628422.2) and gentamicin (GenBank accession number:AJ628149.4). The primers Pkanjk-up1 and Pkanjk-down1 were used to amplify a $1.95 \mathrm{~kb}$ fragment containing intact $k a n \mathrm{~J}$ and $k a n \mathrm{~K}$. The PCR product was digested with HindIII and BamHI and then ligated to pSPU241; thus, pJK241 was generated. The $2.6 \mathrm{~kb}$ insert containing PermE*-kanJK-To was recovered as a $B g l \mathrm{II}$ fragment and then inserted into the same site of pEAP1 to generate PSPUJK1.

The primers Ph1 and Ph2 were used to amplify the downstream homologous arm and to generate a gene replacement vector with kanJK genes under PermE*. The primers $\mathrm{Ph} 3$ and $\mathrm{Ph} 4$ were utilized to amplify the 

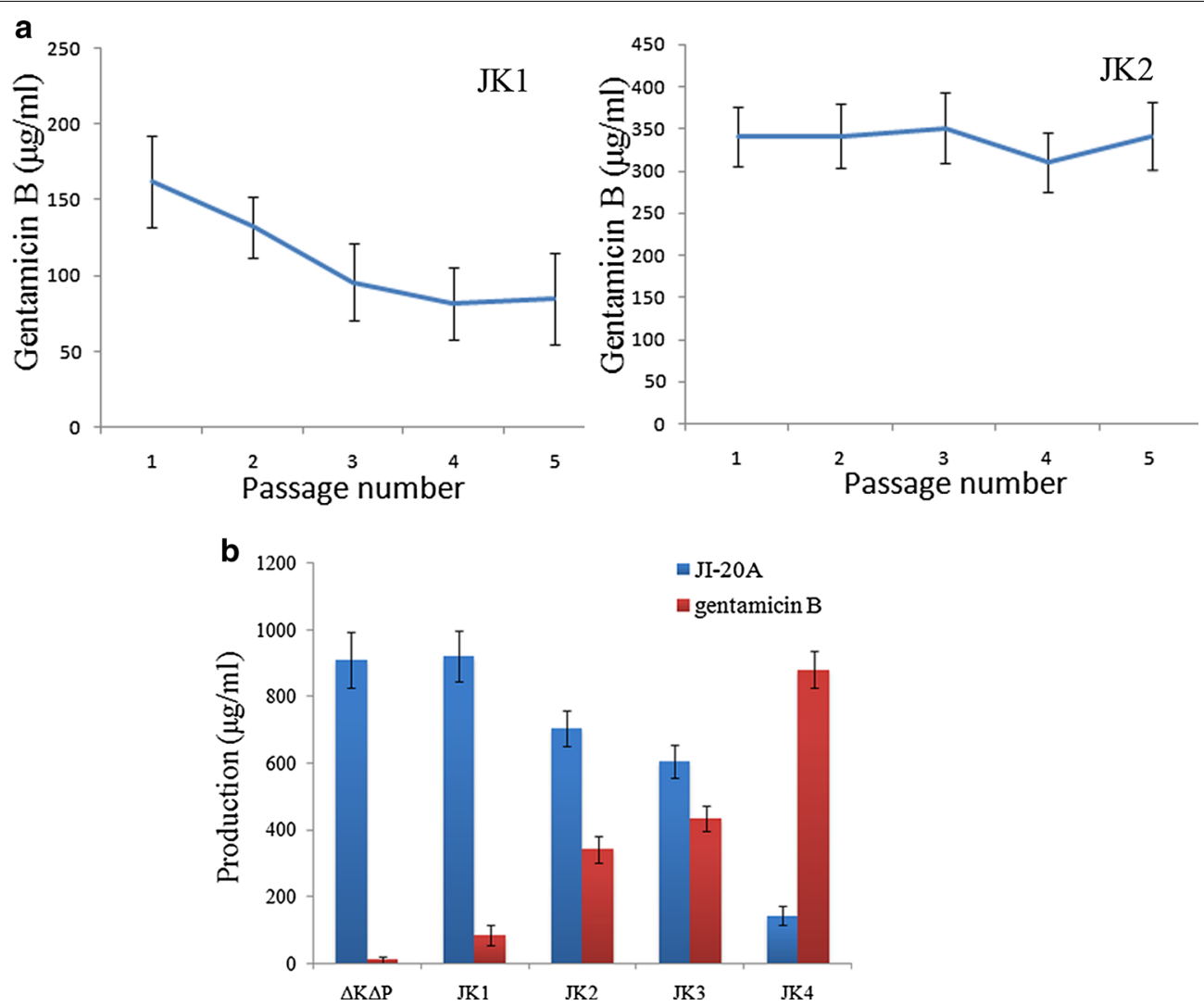

Fig. 5 Production and stability analysis of gentamicin B-producing strains. a Stability of gentamicin B production in M. echinosporaJK1 and JK2 strains. b Production analysis of gentamicin B in M. echinospora $\triangle \mathrm{K} \triangle \mathrm{P}$ and kanJK heterologous expression strains. Error bars represent standard deviations. Samples were analyzed through HPLC and quantified on the basis of the peak areas

upstream homologous arm. Pkanjk-up2 and Pkanjkdown2 were also employed to amplify the intact kanjK fragment. Perm-up and Perm-down were used to amplify PermE*. PermE*, kanJK, and the upstream homologous arm fragment were fused through overlap extension PCR in accordance with a previously described procedure [24]. The overlapping PCR product was ligated with pMD18-T (Takara) and digested with $X b a \mathrm{I}$ and SmaI; the downstream homologous arm was digested with $\mathrm{XbaI}$ and SmaI. Afterward, the digested fragments were ligated to pKC1139; thus, pSPUJK2 was generated.

The primers $\mathrm{Ph} 1$ and $\mathrm{Ph} 2$ were used to amplify the downstream homologous arm and the primers $\mathrm{Ph} 33$ and $\mathrm{Ph} 4$ were utilized to amplify the upstream homologous arm to generate a vector for gene replacement. Pkanjkup3 and Pkanjk-down2 were also used to amplify kanJK. The overlap extension PCR was employed using the primers $\mathrm{Ph} 4$ and Pkanjk-down2 to fuse the kanJK fragment and the upstream homologous arm. The overlap PCR product was ligated using $\mathrm{PMD} 18-\mathrm{T}$ (Takara) and then digested with $X b a \mathrm{I}$ and SmaI. The downstream homologous arm was digested with $X b a \mathrm{I}$ and SmaI. The digested fragments were then ligated to pKC1139; thus, pSPUJK3 was generated.

The primers $\mathrm{Ph} 34$ and $\mathrm{Ph} 4$ were used to amplify the upstream homologous arm to generate a gene replacement vector with $k a n J K$ genes under the strong promoter PhrdB. Pkanjk-up4 and Pkanjk-down2 were utilized to amplify the intact kanJK fragment. Phrd-up and Phrddown were also used to amplify $\mathrm{P} h r d \mathrm{~B}$. The upstream homologous arm fragment, $\mathrm{PhrdB}$, and kanJK were then fused through overlap extension PCR. The overlap PCR product was then ligated with $\mathrm{pMD} 18-\mathrm{T}$ and then digested with $X b a \mathrm{I}$ and SmaI. The downstream homologous arm was digested with $\mathrm{XbaI}$ and SmaI. The digested fragments were ligated to $\mathrm{pKC} 1139$. Thus, pSPUJK4 was generated.

\section{Construction of genK-disrupting and kanJK-expressing strains}

genK-disrupting and kanJK-expressing plasmids were introduced to $M$. echinospora through conjugation on MS medium at $28{ }^{\circ} \mathrm{C}$ for $24 \mathrm{~h}$. After the medium was 
Table 2 Strains and plasmids used in this study

\begin{tabular}{|c|c|c|}
\hline Strains or plasmids & Relevant characteristic & Reference or source \\
\hline \multicolumn{3}{|l|}{ Strains } \\
\hline E. coliTOP10 & 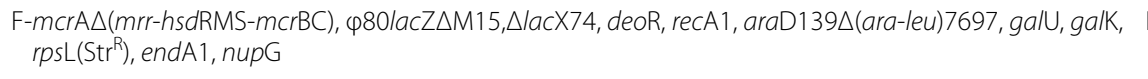 & Invitrogen \\
\hline $\begin{array}{l}\text { E. coli ET12567/ } \\
\text { pUZ8002 }\end{array}$ & Methylation defective, strain used in E. coli-streptomyces intergeneric conjugation & [25] \\
\hline S. kanamyceticus & Kanamycin producing strain & CGMCC4.1441 \\
\hline M. echinospora & Wild-type strain, gentamicin C1a, C2, C2a, and C1 producer & ATCC 15835 \\
\hline M. echinospora $\triangle P$ & M. echinospora with disrupted genP & [7] \\
\hline M. echinospora $\Delta \mathrm{K} \triangle \mathrm{P}$ & M. echinospora with disrupted genK and genP & This study \\
\hline M. echinospora JK1 & $\begin{array}{l}\text { Heterologous, genome-based expression of kanJ and kanK, with replacement of the native promoter } \\
\text { by PermE* in M. echinospora } \Delta \mathrm{K} \triangle \mathrm{P} \text {. }\end{array}$ & This study \\
\hline M. echinospora JK2 & $\begin{array}{l}\text { M. echinospora } \triangle K \triangle P+\text { heterologous expression of kanJ and kanK under the promoter PermE*, inser- } \\
\text { tion at genP locus in M. echinospora } \triangle K \triangle P\end{array}$ & This study \\
\hline M. echinospora JK3 & $\begin{array}{l}\text { Heterologous expression of kanJ and kanK under promoter PgenP, insertion at genP locus in M. echinos- } \\
\text { pora } \triangle \mathrm{K} \triangle \mathrm{P}\end{array}$ & -This study \\
\hline M. echinospora JK4 & $\begin{array}{l}\text { Heterologous expression of kanJ and kanK under promoter PhrdB, insertion at genP locus in M. echinos- } \\
\text { pora } \Delta K \Delta \mathrm{P}\end{array}$ & -This study \\
\hline \multicolumn{3}{|l|}{ Plasmids } \\
\hline pKC1139 & E. coli-streptomyces shuttle vector, $\mathrm{Am}^{\mathrm{R}}$ & [26] \\
\hline pSPU241 & plJ2925 derivative carrying the Streptomyces constitutive promoter PermE* and To terminator, Amp ${ }^{R}$ & [8] \\
\hline pEAP1 & $\begin{array}{l}\text { pSET152 carrying ermE, the apramycin resistance-conferring gene aac(3)IV was replaced by the ampi- } \\
\text { cillin resistance-conferring gene bla, } \mathrm{Amp}^{R}, \mathrm{Erm}^{\mathrm{R}}\end{array}$ & [8] \\
\hline pSPU503 & pKC1139 carrying homologous arms of genK (gacD), used in genK disruption & [12] \\
\hline pJK1 & pEAP1 carrying PermE*-kanJ-kanK, used in generating M. echinospora JK1 & This study \\
\hline pJK2 & pKC1139 carrying homologous arms and kanJK, used in generating M. echinospora JK2 & This study \\
\hline pJK3 & pKC1139 carrying homologous arms and PermE*-kanJ-kanK, used in generating M. echinospora JK3 & This study \\
\hline pJK4 & pKC1139 carrying homologous arms andPhrdB-kanJ-kanK, used in generating M. echinospora JK4 & This study \\
\hline
\end{tabular}

$A m p^{R}$ ampicillin resistance, $A m^{R}$ ampramycin resistance, $E r m^{R}$ erythromycin resistance

spread with $50 \mathrm{mg}$ of apramycin (erythromycin was used for pSPUJK1) and $100 \mathrm{mg}$ of pipemidic acid per liter, incubation was performed at $28{ }^{\circ} \mathrm{C}$ for 7 days. Exconjugants were initially selected to determine the apramycin-resistance (the first crossover event) phenotype because pSPU503, pSPUJK2, pSPUJK3, and pSPUJK4 plasmids contain an apramycin-resistance gene; the apramycin-sensitive (the second crossover event) phenotype was then used to isolate the strains via the desired double-crossover homologous recombination event. Genomic DNA was extracted and used as a template DNA in PCR. The PCR products were subjected to DNA sequencing to demonstrate that $k a n \mathrm{~J}$ and $k a n \mathrm{~K}$ exist in the kanJK-expressing strains.

\section{Antibiotic isolation and analysis}

The $\mathrm{pH}$ of the culture broth was adjusted to 2.0 by using $\mathrm{H}_{2} \mathrm{SO}_{4}$. The acidified broth was agitated for $30 \mathrm{~min}$ and then centrifuged at $11,378 \times g 10 \mathrm{~min}$. The $\mathrm{pH}$ of the supernatant was readjusted to 7.0 with $\mathrm{NaOH}$. The pretreated supernatant was centrifuged again at $11,378 \times g$ for $10 \mathrm{~min}$. The supernatant was then applied to strongly acidic resin $001 \times 7$ (Shandong Lukang Record Pharmaceutical Co., Ltd).The bound substances were eluted with $2 \mathrm{~mol} / \mathrm{L} \mathrm{NH}_{4} \mathrm{OH}$. Second cation-exchange chromatography was performed on weakly acidic resin D152 (Shandong Lukang Record Pharmaceutical Co., Ltd). The bound substances were eluted with the gradient elution of $\mathrm{NH}_{4} \mathrm{OH}$ (from 0.1 to $1.0 \mathrm{~mol} / \mathrm{L}$ ).

The elution from the acidic resin was used as the sample for the reversed-phase HPLC-ELSD analysis in a reverse $\mathrm{C} 18$ column at an evaporation temperature of $45{ }^{\circ} \mathrm{C}$, nitrogen pressure of $3.5 \mathrm{bar}$, and a mobile phase of $0.2 \mathrm{~mol} / \mathrm{L}$ trifluoroacetatic acid-methanol (97:3) at a flow rate of $0.6 \mathrm{ml} / \mathrm{min}$. Authentic gentamicin B was used as standard. The purified products were analyzed using an LC/MS/MS instrument (Bruker micrOTOF-Q). The mass spectrometer was set in a positive mode. ${ }^{1} \mathrm{H}$ and ${ }^{13} \mathrm{C}$ NMR data were recorded on Bruker AV600 at $600 \mathrm{MHz}$ frequency, and $\mathrm{D}_{2} \mathrm{O}$ was used as a solvent. 


\section{Additional file}

Additional file 1: Figure S1. Schematic representation and confirmation of recombination strains. (A) Gene disruption of genK and genP. (B) Expression of kanJ and kanK by site specific intergration. (C) Expression of kanJ and kanK with the promoter PermE*. (D) Expression of kanJ and kanK with the native promoter of genP. (E) Expression of kanJ and kanK with promoter PhrdB

Additional file 2: Figure S2. ${ }^{1} \mathrm{H}$ NMR spectrum of the new compound from kanJK expression strains.

Additional file 3: Table S1. Sequence of primers used in this study.

\section{Authors' contributions}

$\mathrm{XN}$ performed the experiments, analyzed the primary data, and prepared the manuscript. ZS constructed M. echinospora JK3 and JK4 strains and assisted in fermentation experiments. YG assisted in the MS and NMR data analysis and revised the manuscript. $\mathrm{HC}$ assisted in product purification. $\mathrm{HX}$ supervised the whole research and revised the manuscript. All authors read and approved the final manuscript.

\section{Acknowledgements}

This work was supported by the National Natural Science Foundation of China (8127341), Specialized Research Fund for the Doctoral Program of Higher Education (20122134130001), and the General Project of Scientific Research of the Education Department of Liaoning Province (L2014389).

\section{Competing interests}

The authors declare that they have no competing interests.

Received: 13 October 2015 Accepted: 22 December 2015

Published online: 05 January 2016

\section{References}

1. Petrikkos G, Giamarellou H, Tsagaraki C, Pefanis A. Evaluation of the efficacy and safety of isepamicin in the treatment of various bacterial infections. J Chemother. 1995;7(Suppl 2):161-4.

2. Testa RT, Tilley BC. Biotransformation, a new approach to aminoglycoside biosynthesis. II. Gentamicin. J Antibiot. 1976;29(2):140-6.

3. Kharel MK, Basnet DB, Lee HC, Liou K, Moon YH, Kim JJ, Woo JS, Sohng JK. Molecular cloning and characterization of a 2-deoxystreptamine biosynthetic gene cluster in gentamicin-producing Micromonospora echinospora ATCC15835. Mol Cells. 2004;18(1):71-8.

4. Unwin J, Standage S, Alexander D, Hosted T Jr, Horan AC, Wellington EM Gene cluster in Micromonospora echinospora ATCC15835 for the biosynthesis of the gentamicin C complex. J Antibiot. 2004;57(7):436-45.

5. Park JW, Hong JSJ, Parajuli N, Jung WS, Park SR, Lim SK, Sohng JK, Yoon YJ. Genetic dissection of the biosynthetic route to gentamicin A2 by heterologous expression of its minimal gene set. Proc Natl Acad Sci USA. 2008;105(24):8399-404.

6. Huang C, Huang F, Moison E, Guo J, Jian X, Duan X, Deng Z, Leadlay $P F$, Sun Y. Delineating the biosynthesis of gentamicin X2, the common precursor of the gentamicin C antibiotic complex. Chem Biol. 2015;22(2):251-61.

7. Guo J, Huang F, Huang C, Duan X, Jian X, Leeper F, Deng Z, Leadlay PF, Sun Y. Specificity and promiscuity at the branch point in gentamicin biosynthesis. Chem Biol. 2014;21(5):608-18.
8. Ni X, Sun Z, Zhang H, He H, Ji Z, Xia H. Genetic engineering combined with random mutagenesis to enhance G418 production in Micromonospora echinospora. J Ind Microbiol Biotechnol. 2014;41(9):1383-90.

9. GuY, Ni X, Ren J, Gao H, Wang D, Xia H. Biosynthesis of epimers C2 and C2a in the gentamicin C complex. ChemBioChem. 2015;16(13):1933-42.

10. Hong $W$, Yan $L$. Identification of gntK, a gene required for the methylation of purpurosamine C-6' in gentamicin biosynthesis. J Gen Appl Microbiol. 2012;58(5):349-56.

11. Karki S, Kim JY, Park SH, Kwon HJ. Gene inactivation study on gntK, a putative C-methyltransferase gene in gentamicin biosynthesis from Micromonospora echinospora. J Korean Soc Appl Biol Chem. 2012;55(3):1-4.

12. Li D, Li H, Ni X, Zhang H, Xia H. Construction of a gentamicin C1a-overproducing strain of Micromonospora purpurea by inactivation of the gacD gene. Microbiol Res. 2013;168(3):263-7.

13. Shao L, Chen J, Wang C, Li J, Tang Y, Chen D, Liu W. Characterization of a key aminoglycoside phosphotransferase in gentamicin biosynthesis. Bioorg Med Chem Lett. 2013;23(5):1438-41.

14. Sucipto $H$, Kudo F, Eguchi T. The last step of kanamycin biosynthesis: unique deamination reaction catalyzed by the alpha-ketoglutaratedependent nonheme iron dioxygenase KanJ and the NADPH-dependent reductase KanK. Angew Chem Int Ed Engl. 2012;51(14):3428-31.

15. Zupančič-Kralj Lucija. Identification of gentamicin impurities by liquid chromatography tandem mass spectrometry. JPharm Biomed Anal. 2009;50(5):1037-43.

16. Bérdy J, Pauncz JK, Vajna ZM, Horváth G, Gyimesi J, Koczka I. Metabolites of gentamicin-producing Micromonospora species. I. Isolation and identification of metabolites. J Antibiot. 1977;30(11):945-54.

17. Nagabhushan TL, Cooper AB, Tsai H, Daniels PJL, Miller GH. The syntheses and biological properties of 1-N-(S-4-amino-2-hydroxybutyryl)gentamicin B and 1-N-(S-3-amino-2-hydroxypropionyl)-gentamicin B. J Antibiot. 1978;31(7):681-7.

18. Combes $\mathrm{P}$, Till R, Bee S, Smith MC. The streptomyces genome contains multiple pseudo-attB sites for the (phi)C31-encoded site-specific recombination system. J Bacteriol. 2002;184(20):5746-52.

19. Salis HM, Mirsky EA, Voigt CA. Automated design of synthetic ribosome binding sites to control protein expression. Nat Biotechnol. 2009;27(10):946-50.

20. Du D, Zhu Y, Wei J, Tian Y, Niu G, Tan H. Improvement of gougerotin and nikkomycin production by engineering their biosynthetic gene clusters. Appl Microbiol Biotechnol. 2013;97(14):6383-96.

21. Ōmura S, Ikeda H, Ishikawa J, Hanamoto A, Takahashi C, Shinose M, Takahashi Y, Horikawa H, Nakazawa H, Osonoe T. Genome sequence of an industrial microorganism Streptomyces avermitilis: deducing the ability of producing secondary metabolites. Proc Natl Acad Sci USA. 2001;98(21):12215-20

22. Keasling JD. Synthetic biology and the development of tools for metabolic engineering. Metab Eng. 2012;14(3):189-95.

23. Sambrook J, Russell DW. Molecular cloning: a laboratory manual. 3rd ed. New York: Cold spring harbor laboratory press; 2001.

24. Shevchuk NA, Bryksin AV, Nusinovich YA, Cabello FC, Sutherland M, Ladisch S. Construction of long DNA molecules using long PCRbased fusion of several fragments simultaneously. Nucleic Acids Res. 2004;32(2):e19-19.

25. MacNeil DJ, Gewain KM, Ruby CL, Dezeny G, Gibbons PH, MacNeil T. Analysis of Streptomyces avermitilis genes required for avermectin biosynthesis utilizing a novel integration vector. Gene. 1992;111(1):61-8.

26. Bierman M, Logan R, O'Brien K, Seno ET, Rao RN, Schoner BE. Plasmid cloning vectors for the conjugal transfer of DNA from Escherichia coli to Streptomyces spp. Gene. 1992;116(1):43-9. 